\title{
Factors predictive of acute renal failure and need for hemodialysis among ED patients with rhabdomyolysis
}

\author{
William G. Fernandez MD, MPH ${ }^{a, b, *}$, Oliver Hung MDc, G. Richard Bruno MD ${ }^{d}$, \\ Sandro Galea MD, MPH, DrPH ${ }^{e}$, William K. Chiang MD ${ }^{a}$
}

\author{
${ }^{a}$ Department of Emergency Medicine, New York University/Bellevue Hospital Center, NY 10016, USA \\ ${ }^{\mathrm{b}}$ Department of Emergency Medicine, Boston University School of Medicine, Boston Medical Center, Boston, MA 02118, USA \\ ${ }^{\mathrm{c}}$ Department of Emergency Medicine, Morristown Memorial Hospital, Morristown, NJ 07960, USA \\ ${ }^{\mathrm{d} D e p a r t m e n t ~ o f ~ E m e r g e n c y ~ M e d i c i n e, ~ S t a t e ~ U n i v e r s i t y ~ o f ~ N e w ~ Y o r k ~ D o w n s t a t e, ~ K i n g s ~ C o u n t y ~ H o s p i t a l ~ C e n t e r, ~}$ \\ Brooklyn, NY 11203, USA \\ ${ }^{\mathrm{e}}$ Center for Urban Epidemiologic Studies, New York Academy of Medicine, New York, NY 10029, USA
}

Received 22 August 2003; accepted 23 November 2003

\begin{abstract}
Objective: We assessed the primary causes of rhabdomyolysis, the factors associated with the development of acute renal failure (ARF), and the need for hemodialysis (HD) among a series of patients presenting to an urban emergency department with rhabdomyolysis.

Methods: A chart review between January 1992 and December 1995 was conducted of patients aged 18 years or older with a diagnosis of rhabdomyolysis and an initial serum creatine phosphokinase greater than $1000 \mathrm{U} / \mathrm{L}$. Patients were excluded if they had evidence of myocardial ischemia, cerebrovascular insufficiency, or the development of rhabdomyolysis after hospitalization. Demographic information, presumed causative factors, past medical history, medication usage, and laboratory data were collected. Results: Ninety-seven patients (93 men, 4 women) were enrolled, with a mean age of 35.7 years. The most common causes of rhabdomyolysis were cocaine (30), exercise (29), and immobilization (18). Seventeen of 97 (17.5\%) patients developed ARF; 8 patients $(8.25 \%)$ needed HD. Several clinical and laboratory factors were statistically associated with development of ARF and need for HD. The only variable that was predictive of both ARF and need for HD in separate multivariate regression models was the initial creatinine $(\mathrm{Cr})$. Initial blood urea nitrogen also was predictive of the need for HD. No patient developed ARF with an initial Cr less than $1.7 \mathrm{mg} / \mathrm{dL}$.

Conclusion: Acute renal failure and need for HD are common complications of rhabdomyolysis. Except for initial serum $\mathrm{Cr}$ and blood urea nitrogen, clinical and laboratory factors were not reliable predictors for the development of ARF or need for HD.

(C) 2005 Elsevier Inc. All rights reserved.
\end{abstract}

* Corresponding author. Department of Emergency Medicine, Boston Medical Center, One Boston Medical Center Place, Dowling 1 South, Boston, MA 02118, USA. Tel.: +1 617414 4927; fax: +1 2124147759 .

E-mail address: william.fernandez@bmc.org (W.G. Fernandez).

\section{Introduction}

Rhabdomyolysis results from injury to skeletal muscle and the resulting release of skeletal muscle contents into the 
systemic circulation. The etiologies of rhabdomyolysis may vary, but the outcome is the same: as membrane integrity is lost, intracellular sodium and calcium levels rise, resulting in cell break down and death [1,2]. In turn, various intramuscular components, including creatine phosphokinase (CPK), are released into plasma. Clinically, an elevated level of CPK is the primary marker used to identify rhabdomyolysis. It is postulated that the breakdown

Table 1 Demographic data of patients with rhabdomyolysis tested via $\chi^{2}$ analysis for development of ARF and need for HD in an ED sample $(\mathrm{N}=97)$

\begin{tabular}{|c|c|c|c|c|c|c|c|c|c|c|}
\hline Characteristic & $\mathrm{N}$ & $\%$ & $\mathrm{ARF}$ & $\% \mathrm{ARF}$ & $\chi^{2}$ & $P$ & $\mathrm{HD}$ & $\% \mathrm{HD}$ & $\chi^{2}$ & $P$ \\
\hline Total & 97 & $100 \%$ & 17 & 17.5 & & & 8 & 8.25 & & \\
\hline \multicolumn{11}{|l|}{ Gender } \\
\hline Male & 93 & 95.9 & 16 & 17.2 & 0.1612 & .69 & 8 & 8.6 & 0.375 & .54 \\
\hline Female & 4 & 4.1 & 1 & 25 & & & 0 & 0 & & \\
\hline \multicolumn{11}{|l|}{ Ethnicity } \\
\hline African American & 43 & 44.3 & 8 & 18.6 & 0.1263 & .72 & 5 & 11.6 & 0.8798 & .35 \\
\hline Latino/Hispanic & 27 & 27.8 & 6 & 22.2 & & & 2 & 7.4 & & \\
\hline Caucasian & 25 & 25.8 & 3 & 12 & & & 1 & 4 & & \\
\hline Other & 2 & 2.1 & 0 & 0 & & & 0 & 0 & & \\
\hline \multicolumn{11}{|l|}{ Age } \\
\hline $18-24$ & 17 & 17.7 & 3 & 17.7 & 0.7647 & .3819 & 1 & 5.8 & 3.13 & .07 \\
\hline $25-34$ & 32 & 33.3 & 4 & 12.5 & & & 1 & 3.1 & & \\
\hline $35-44$ & 29 & 30.2 & 5 & 17.2 & & & 3 & 10.3 & & \\
\hline $45-54$ & 9 & 9.4 & 2 & 22.2 & & & 0 & 0 & & \\
\hline $55-64$ & 3 & 3.1 & 2 & 66.7 & & & 2 & 66.7 & & \\
\hline $65+$ & 6 & 6.3 & 1 & 16.7 & & & 1 & 16.7 & & \\
\hline \multicolumn{11}{|l|}{ Hypertension } \\
\hline Yes & 10 & 10.3 & 3 & 30 & 1.2 & .27 & 3 & 30 & 6.9717 & .0083 \\
\hline No & 87 & 89.7 & 14 & 16.1 & & & 5 & 5.75 & & \\
\hline \multicolumn{11}{|l|}{ Diabetes } \\
\hline Yes & 2 & 2.1 & 0 & 0 & 0.43 & .51 & 0 & 0 & 0.1836 & .67 \\
\hline No & 95 & 97.9 & 17 & 17.9 & & & 8 & 8.4 & & \\
\hline \multicolumn{11}{|l|}{ Renal disorder } \\
\hline Yes & 4 & 4.1 & 2 & 50 & 3.04 & .08 & 2 & 50 & 9.6112 & .0019 \\
\hline No & 93 & 95.9 & 15 & 16.1 & & & 6 & 6.45 & & \\
\hline \multicolumn{11}{|l|}{$H I V$} \\
\hline Yes & 17 & 17.5 & 5 & 29.4 & 2.01 & .16 & 2 & 11.8 & 0.337 & .56 \\
\hline No & 80 & 82.5 & 12 & 15 & & & 6 & 7.5 & & \\
\hline \multicolumn{11}{|l|}{ Pain in legs/back } \\
\hline Yes & 46 & 47.4 & 6 & 13 & 1.22 & .27 & 2 & 4.4 & 1.758 & .18 \\
\hline No & 51 & 52.6 & 11 & 21.5 & & & 6 & 11.8 & & \\
\hline \multicolumn{11}{|l|}{ Dark urine } \\
\hline Yes & 35 & 36.1 & 4 & 11.4 & 1.4 & .24 & 2 & 5.7 & 0.4643 & .5 \\
\hline No & 62 & 63.9 & 13 & 21 & & & 6 & 9.7 & & \\
\hline \multicolumn{11}{|l|}{ Incarcerated } \\
\hline Yes & 38 & 39.2 & 5 & 13.2 & 0.82 & .36 & 2 & 5.3 & 0.7353 & .39 \\
\hline No & 59 & 60.82 & 12 & 20.3 & & & 6 & 10.2 & & \\
\hline \multicolumn{11}{|c|}{ Psychiatric disorder } \\
\hline Yes & 11 & 11.3 & 1 & 9.1 & 0.61 & .43 & 0 & 0 & 1.11 & .29 \\
\hline No & 86 & 88.7 & 16 & 18.6 & & & 8 & 9.3 & & \\
\hline
\end{tabular}


products of myoglobin may have a directly toxic effect on the renal system [3]. This potentially leads to renal complications of rhabdomyolysis [4].

One of the first accounts to describe the clinical manifestations of rhabdomyolysis (the triad of muscle pain, weakness, and tea-colored urine) was in the German literature at the beginning of the 20th century [5]. A number of reports since have described the array of etiologic factors that can lead to the condition [6-23]. These can be grouped into the following categories: intrinsic muscle insults/trauma, drugs/toxins, metabolic disorders, hypoxia/ischemia, thermal regulation disorders, and infection. Alcohol, drugs, infection, trauma, and seizures have been cited as the most common causes of rhabdomyolysis [6]. The first report to document the relationship between rhabdomyolysis and the complications of acute renal failure (ARF) and death was published during the London Blitz during World War II [24]. Since then, rhabdomyolysis has been reported to cause up to $7 \%$ of all cases of ARF [3]. The incidence of ARF as a result of rhabdomyolysis has been reported to range from $0 \%$ to $67 \%$ in various clinical settings [25-30], depending on the inciting cause. Furthermore, another study reported that in patients treated for ARF resulting from rhabdomyolysis, nearly $4 \%$ ultimately required hemodialysis (HD) [6].

To date, there is a paucity of emergency department (ED)-based research specifically studying rhabdomyolysis and the factors on presentation to the ED that predict subsequent development of ARF or need for HD $[15,16]$. The purpose of this study was to identify the major causes of rhabdomyolysis in patients presenting to an ED setting and to identify the factors that were most predictive of ARF and need for HD in these patients.

\section{Methods}

We conducted a retrospective chart review of ED and inpatient medical records between January 1992 and December 1995 at a university teaching hospital in New York City. The annual number of adult ED patient visits is approximately 90000 per year. Data were collected by one of the authors and annotated onto a standardized data entry sheet. This information was later entered into a computerized database in which no patient identifiers were used. Patients were included in the study if they were aged 18 years or older, had a discharge diagnosis of rhabdomyolysis (ICD-9 code 728.89), and had an initial CPK level drawn in the ED of greater than $1000 \mathrm{U} / \mathrm{L}$. This cutoff was used because it is the degree of CPK elevation that generally indicates a clinically meaningful degree of rhabdomyolysis [28]. Acute renal failure was defined as an acute loss of renal function with a persistent elevation of creatinine $(\mathrm{Cr})$ of greater than or equal to $2.5 \mathrm{mg} / \mathrm{dL}$, which did not improve with hydration [28]. This definition was to distinguish ARF patients from those with chronic renal insufficiency, as well as those suffering from prerenal azotemia. Patients were excluded if there was evidence of myocardial infarction, cerebrovascular accident, or if rhabdomyolysis developed after admission to the hospital as a result of a coexisting condition or iatrogenic complication.

Information about factors that were potentially causative for rhabdomyolysis or predictive for progression to ARF was abstracted from the medical records of patients meeting inclusion criteria for this study. Data were collected on demographic characteristics, past medical history (hypertension, diabetes, history of renal insufficiency, HIV/AIDS), medication use, recent physical exertion, recent trauma (crush injury, motor vehicle collision, fall, assault, burn), seizure, immobilization, dehydration, viral/bacterial illness, metabolic derangements (diabetic/alcoholic ketoacidosis), hyper- or hypothermia, or illicit drug use. The presenting signs and symptoms of patients were recorded, including presence of localized pain, history of dark urine, or neurological findings such as weakness or altered mental status. The presence of hyper- or hypotension on the initial triage report (defined as systolic blood pressure more than 150 or less than 90 , respectively) was collected.

We used a standard statistical software package (The SAS System for Windows, Version 8) to analyze our data. To identify individual correlates of ARF and need for HD, we carried out $\chi^{2}$ analysis on sociodemographic variables and Student $t$ testing on the mean scores of laboratory data. For the multivariate logistic regression analyses, we identified a set of factors a priori that we thought were likely predictors of the 2 main study outcomes. A final model predicting renal failure was generated using the backward stepwise elimination technique. Overall, we sought to identify the association between patients' clinical and laboratory findings, and the endpoints of ARF and need for HD, while controlling for relevant demographic variables. An institutional review board approved this project.

\section{Results}

The authors reviewed 166 medical records of patients discharged during the period of January 1992 to December 1995 for possible enrollment into the study. Eighty-six patients' charts were excluded (37 had CPK levels less than

Table 2 Causes of rhabdomyolysis cases, ARF, and need for HD in an emergency-department sample $(\mathrm{N}=97)$

\begin{tabular}{lrrlllc}
\hline Causative factor & $\mathrm{N}$ & $\%$ & $\mathrm{ARF}$ & $\%$ & $\mathrm{HD}$ & $\%$ \\
\hline Cocaine & 30 & 30.9 & 6 & 20 & 3 & 10 \\
Exercise & 29 & 29.9 & 3 & 10.3 & 1 & 3.5 \\
Immobilization & 18 & 18.6 & 3 & 16.7 & 2 & 11.1 \\
Ethanol withdrawal & 4 & 4.1 & 1 & 25 & 1 & 25 \\
Seizure & 4 & 4.1 & 2 & 50 & 1 & 25 \\
Trauma & 4 & 4.1 & 1 & 25 & 0 & 0 \\
Other & 8 & 8.2 & 1 & 12.5 & 0 & 0 \\
\hline
\end{tabular}


Table 3 Associations between mean laboratory values and ARF and need for HD via Student $t$ testing $(\mathrm{N}=97)$

\begin{tabular}{|c|c|c|c|c|c|c|c|c|}
\hline Characteristic & $\mathrm{ARF}$ & Mean & $\mathrm{SD}$ & $P$ & HD & Mean & $\mathrm{SD}$ & $P$ \\
\hline \multirow[t]{2}{*}{ SBP } & $\mathrm{Y}$ & 130.5 & 33.6 & .45 & $\mathrm{Y}$ & 135.00 & 33.91 & .45 \\
\hline & $\mathrm{N}$ & 127 & 23.2 & & $\mathrm{~N}$ & 127.04 & 24.45 & \\
\hline \multirow[t]{2}{*}{ HR } & $\mathrm{Y}$ & 106.06 & 32.6 & .18 & $\mathrm{Y}$ & 99.33 & 31.1 & .9 \\
\hline & $\mathrm{N}$ & 96.3 & 25.8 & & $\mathrm{~N}$ & 97.93 & 27.05 & \\
\hline \multirow[t]{2}{*}{ TEMP } & $\mathrm{Y}$ & 100.56 & 3.25 & .97 & $\mathrm{Y}$ & 100.88 & 3.15 & .77 \\
\hline & $\mathrm{N}$ & 100.54 & 2.9 & & $\mathrm{~N}$ & 100.52 & 2.95 & \\
\hline \multirow[t]{2}{*}{ CPK1 } & $\mathrm{Y}$ & 24019 & 27770 & .26 & $\mathrm{Y}$ & 19863 & 19799 & .14 \\
\hline & $\mathrm{N}$ & 33559 & 44883 & & $\mathrm{~N}$ & 32968 & 43791 & \\
\hline \multirow[t]{2}{*}{ CPK2 } & $\mathrm{Y}$ & 38092 & 44120 & .65 & $\mathrm{Y}$ & 49502 & 53371 & .75 \\
\hline & $\mathrm{N}$ & 44744 & 57091 & & $\mathrm{~N}$ & 43046 & 55290 & \\
\hline \multirow[t]{2}{*}{ K1 } & $\mathrm{Y}$ & 4.65 & 1.07 & .25 & $\mathrm{Y}$ & 4.71 & 1.19 & .43 \\
\hline & $\mathrm{N}$ & 4.33 & 0.7 & & $\mathrm{~N}$ & 4.36 & 0.73 & \\
\hline \multirow[t]{2}{*}{$\mathrm{CO} 2$} & $\mathrm{Y}$ & 17.25 & 3.4 & .0001 & $\mathrm{Y}$ & 16.79 & 3.07 & .0006 \\
\hline & $\mathrm{N}$ & 24 & 4.87 & & $\mathrm{~N}$ & 23.37 & 5.13 & \\
\hline \multirow[t]{2}{*}{ BUN } & $\mathrm{Y}$ & 63.47 & 51.07 & .0027 & $\mathrm{Y}$ & 66.5 & 46.21 & .034 \\
\hline & $\mathrm{N}$ & 19.37 & 15.78 & & $\mathrm{~N}$ & 23.56 & 26.15 & \\
\hline \multirow[t]{2}{*}{$\mathrm{Cr} 1$} & $\mathrm{Y}$ & 5.79 & 3.34 & .0001 & $\mathrm{Y}$ & 6.86 & 3.46 & .0037 \\
\hline & $\mathrm{N}$ & 1.31 & 0.44 & & $\mathrm{~N}$ & 1.66 & 1.48 & \\
\hline \multirow[t]{2}{*}{$\mathrm{Ca}$} & $\mathrm{Y}$ & 7.98 & 1.21 & .0065 & $\mathrm{Y}$ & 8.3 & 1.53 & .059 \\
\hline & $\mathrm{N}$ & 9.33 & 0.7 & & $\mathrm{~N}$ & 9.15 & 0.87 & \\
\hline \multirow[t]{2}{*}{ SG } & $\mathrm{Y}$ & 1.0181 & 0.0083 & .77 & $\mathrm{Y}$ & 1.0213 & 0.0064 & .38 \\
\hline & $\mathrm{N}$ & 1.0192 & 0.0075 & & $\mathrm{~N}$ & 1.0189 & 0.0078 & \\
\hline \multirow[t]{2}{*}{$\mathrm{PH}$} & $\mathrm{Y}$ & 5.714 & 1.28 & .99 & $\mathrm{Y}$ & 5.75 & 1.07 & .91 \\
\hline & $\mathrm{N}$ & 5.718 & 0.82 & & $\mathrm{~N}$ & 5.71 & 0.9 & \\
\hline \multirow{2}{*}{ Urine dip } & $\mathrm{Y}$ & 2.5 & 0.63 & .0016 & $\mathrm{Y}$ & 2.88 & 0.35 & .0042 \\
\hline & $\mathrm{N}$ & 1.79 & 1.14 & & $\mathrm{~N}$ & 1.83 & 1.1 & \\
\hline
\end{tabular}

Abbreviations: SBP, systolic blood pressure; HR, heart rate; K1, initial potassium; SG, urine specific gravity; PH, urine pH.

$1000 \mathrm{U} / \mathrm{L} ; 19$ had rhabdomyolysis develop only after admission as a result of another disorder; 9 cases of rhabdomyolysis occurred in patients younger than 18 years; and 4 were mistakenly assigned an ICD-9 code of 728.89). The remaining 97 patients' charts were included into the study. The mean initial CPK for the sample was 31886 ; the mean peak CPK was 43578 . Demographic characteristics of the sample are presented in Table 1. The mean age of patients was 35.7 years. Nearly $75 \%$ were minorities, whereas almost all patients in this sample were men. Emergency department treatment for all patients included intravenous hydration and sodium bicarbonate infusion. Table 2 summarizes the major causes of rhabdomyolysis of patients in this study. The most common causes of rhabdomyolysis in this sample were cocaine $(\mathrm{n}=30)$, exercise $(n=29)$, and immobilization $(n=18)$. Direct traumatic injury, seizure, and ethanol withdrawal each had caused 4 cases. Seventeen (17.53\%) patients in this series developed ARF, as defined by a persistent elevation in $\mathrm{Cr}$ greater than $2.5 \mathrm{mg} / \mathrm{dL}$. Eight $(8.25 \%)$ of these patients required HD. Two deaths occurred in this sample: in one man (in ARF) died because of prolonged immobilization, and another man (not in ARF) because of cocaine.

Table 3 shows bivariate relations between the demographic data and the development of ARF and HD. There was no difference noted between patients who developed renal failure and those who did not with respect to age, sex, presumptive cause of rhabdomyolysis, prisoner status,

Table 4 Regression model with explanatory variables predicting development of ARF and need for HD (N = 97)

\begin{tabular}{|c|c|c|c|c|c|c|c|c|}
\hline \multirow[t]{2}{*}{ Variable } & \multicolumn{4}{|c|}{$\mathrm{ARF}$} & \multicolumn{4}{|c|}{ HD } \\
\hline & $\beta$ & SE & $\chi^{2}$ & $P$ & $\beta$ & $\mathrm{SE}$ & $\chi^{2}$ & $P$ \\
\hline Intercept & -8.9386 & 5.0310 & 3.1567 & .0756 & -13.1180 & 18.0309 & 0.5293 & .4669 \\
\hline Renal disorder & -0.1652 & 17.6109 & 0.0001 & .9925 & 2.8269 & 7.1951 & 0.1544 & .6944 \\
\hline Hypertension & -5.1852 & 17.8631 & 0.0843 & .7716 & 7.7986 & 9.3017 & 0.7029 & .4018 \\
\hline $\mathrm{CO} 2$ & -0.0451 & 0.1183 & 0.1450 & .7034 & -0.287 & 0.3531 & 0.6643 & .4151 \\
\hline Urine dip for blood & -0.2097 & 0.8192 & 0.0655 & .7980 & 3.7724 & 4.7179 & 0.6393 & .4239 \\
\hline BUN & 0.0489 & 0.0866 & 0.3195 & .5719 & -0.1366 & 0.0618 & 4.8873 & .0271 \\
\hline $\mathrm{Cr}$ & 4.3067 & 1.6138 & 7.1217 & .0076 & 2.3876 & 0.9070 & 6.9292 & .0085 \\
\hline
\end{tabular}




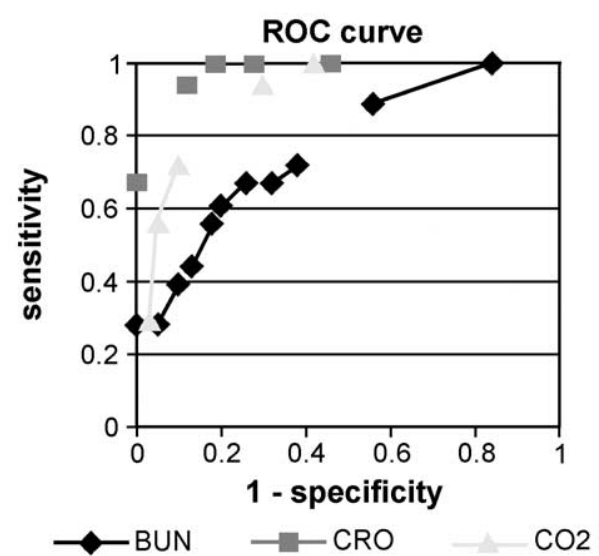

Fig. 1 Receiver operator characteristic (ROC) curve of initial values for blood urea nitrogen (BUN), serum creatinine (CRO), and serum bicarbonate $(\mathrm{CO} 2)$ concentrations.

history of renal disease, history of hypertension, history of diabetes, history of HIV status, history of psychiatric disorder, history of dark urine, or history of leg pain. There were differences between patients who required HD and those who did not in the history of renal disease, as well as history of hypertension.

Additionally, there was no difference between patients who developed ARF and those who did not in urine $\mathrm{pH}$, specific gravity, initial serum potassium, initial CPK level, or peak CPK level. However, patients who developed ARF differed from those who did not in serum bicarbonate $(\mathrm{CO} 2)$ (17.25 vs $24, P<.0001)$, blood urea nitrogen (BUN) $(63.47$ vs $19.37, P=.0027)$, calcium (7.98 vs $9.33, P=.0065)$, initial $\mathrm{Cr}(5.78$ vs $1.31, P<.0001)$, and evidence of blood on urine dipstick $(+2.5 \mathrm{vs}+1.79, P=.0016)$. Patients who required HD differed from those who did not in $\mathrm{CO} 2$ (16.79 vs $23.37, P=.0006$ ), BUN (66.5 vs $23.56, P=.034$ ), initial Cr (6.86 vs $1.66, P=.0037)$, and evidence of blood on urine dipstick $(+2.88$ vs $+1.83, P=.0042)$. All patients in this series that developed ARF or required HD had an abnormal initial serum $\mathrm{Cr}$ level $(\geq 1.7 \mathrm{mg} / \mathrm{dL})$ on presentation to the ED.

Table 4 depicts the separate regression models showing various explanatory variables that attempt to predict the 2 outcome variables. Only the initial $\mathrm{Cr}$ level was a significant predictor of $\operatorname{ARF}(P=.0076)$ in a multivariable linear regression model using backward stepwise elimination. In another regression model, the significant predictors for the need for HD were initial Cr level $(P=.0085)$ and BUN $(P=.027)$.

To show a graphic comparison of the sensitivities and specificities at various levels for each laboratory tests, receiver operator characteristic curves of initial $\mathrm{BUN}, \mathrm{CO}$, and serum $\mathrm{Cr}$ levels for predicting ARF were created. They are illustrated in Fig. 1. Overall, the best curve was for serum $\mathrm{Cr}$, with the highest specificity and lowest false positive rate at a value of $1.7 \mathrm{mg} / \mathrm{dL}$.

\section{Discussion}

Previous research has explored the frequency of ARF among patients with rhabdomyolysis in clinical settings. The prevalence of ARF varies widely depending on the inciting event [6,25-29]. The need for HD as a consequence of rhabdomyolysis, although not generally used as an endpoint, has been reported in one study as less than $4 \%$ [6]. The major diagnostic entities that are reported to lead to rhabdomyolysis include trauma, immobilization, alcohol, drugs, and excessive exercise. In our study of ED patients identified with rhabdomyolysis, the main causative factors were cocaine abuse, exercise, and immobilization. Seventeen $(17.5 \%)$ of these patients developed ARF. Of these, $8(8.25 \%)$ patients went on to require HD-3 because of cocaine abuse, 2 from prolonged immobilization, 1 because of physical exercise, 1 from prolonged seizures, and 1 from severe trauma. One of the 2 deaths in this series was in a patient with prolonged immobilization that had developed ARF. A patient who did not have ARF died from other complications relating to cocaine overdose.

In one study on rhabdomyolysis [28], whereas a history of renal disease was not associated with ARF, there was an association between hypertension and ARF. However, in a final regression model, hypertension was not predictive of ARF [28]. The data from our study support those conclusions. In our study, having a history of either hypertension or of renal disease were not predictive of development of either ARF or HD in a final multivariate logistic regression model.

In previous studies on cocaine-intoxicated patients, the prevalence of $\mathrm{ARF}$ as a consequence of rhabdomyolysis has been reported as $24 \%$ to $33 \%$ [31,32]. Cocaine is suggested to cause ARF by either of 2 processes: vasoconstriction of renal vessels or from the effects of rhabdomyolysis $[33,34]$. In our series, $20 \%$ of the cocaine abusers with rhabdomyolysis developed ARF, whereas $10 \%$ required HD. One patient in our series died from cocaine overdose, although he was not in ARF.

In a series of 35 inmates from Riker's Island with exercise-induced rhabdomyolysis, none developed ARF [29]. In this "pure" form of rhabdomyolysis, it was suggested that ARF does not develop in the absence of metabolic acidosis or hypovolemia. In our study, there were 38 prisoners who developed rhabdomyolysis, of whom 9 were recently arrested by the New York Police Department (NYPD), whereas 29 were New York City Department of Corrections inmates at Riker's Island during the study period. Of the 29 Riker's Island inmates brought to our facility with rhabdomyolysis, 28 were from excessive exercise, and 1 was from trauma. Of the NYPD arrestees that had rhabdomyolysis, 5 were because of cocaine, 2 were because of trauma, and the rest were because of other factors. Overall, 5 prisoners developed ARF: 4 Riker's Island inmates and 1 NYPD arrestee. Of these, only one had evidence of systemic acidosis, whereas the rest had no 
evidence of either metabolic acidosis or hypovolemia. Five prisoners developed ARF: 3 Riker's inmates from excessive exercise, 1 inmate from trauma, and an NYPD arrestee also from trauma. Finally, 2 prisoners required HD: 1 Riker's inmate with exercise-induced rhabdomyolysis and 1 NYPD arrestee with trauma-induced rhabdomyolysis.

Several laboratory values were tested to determine a potential association with development of ARF and need for HD. In our study, the initial $\mathrm{Cr}$ level, bicarbonate, BUN, and urine dipstick for blood had a significant association with both ARF and need for HD in bivariate analyses. The calcium level was shown to be associated with development of ARF, but not needed for HD. In the study by Gabow et al [6], the peak $\mathrm{Cr}$ level, peak potassium level, peak phosphorous level, peak uric acid levels, and trough calcium level were statistically different between those who did and did not develop ARF. Muckart et al [35], in a study of 64 cases of traumatic rhabdomyolysis, sought to predict ARF using the presence of myoglobin in the urine in addition to venous bicarbonate concentration.

In our study, only the initial Cr level was predictive on 2 separate multivariate logistic regression models predicting ARF and need for HD. In fact, no patients presenting with an initial $\mathrm{Cr}$ of less than $1.7 \mathrm{mg} / \mathrm{dL}$ developed ARF. In addition, BUN was predictive of the need for HD. In contrast, Ward [28] reported that although serum $\mathrm{Cr}$ and BUN levels were not predictive, elevated levels of serum CPK, serum potassium, serum phosphorous levels, history on presentation of dehydration, or of sepsis were predictive of ARF in a multivariate logistic regression model. Similarly, a prediction formula $[R=0.7$ (potassium) +1.1 (creatine) +0.6 (albumin) - 6.6] developed by Gabow et al [6] was used to distinguish those at low risk for ARF from those at high risk for ARF. In that study, scores of less than 0.1 were associated with a zero prevalence of ARF, whereas those with $R$ scores of greater than or equal to 0.1 had a $41 \%$ prevalence of ARF. As mentioned earlier, Muckart et al [35] sought to predict ARF using venous bicarbonate concentration and urine myoglobin. Unfortunately, not enough patients had serum phosphorous, serum albumin, or urine myoglobin levels collected in our study to warrant using these prediction models.

It has been postulated that serum $\mathrm{Cr}$ can spuriously be elevated early in the course of rhabdomyolysis because of leakage of preformed $\mathrm{Cr}$ from injured striated muscle $[3,6]$. It is thought that this is because of the high concentration of $\mathrm{Cr}$ within myocytes, which is several times that of serum [3]. This early rise in Cr levels in the initial phase of clinical rhabdomyolysis generally resolves in hours after hydration. In our study, we found that no patient with an abnormal $\mathrm{Cr}$ level had rapid improvement with hydration. In lieu of having baseline renal function testing in advance of ED presentation, an abnormal initial $\mathrm{Cr}$ level seemed to predict $\mathrm{ARF}$ and need for HD among our series of patients with rhabdomyolysis.

\section{Limitations and future directions}

There were important limitations in this project. The study design was a retrospective chart review. It was not our aim in this chart review to evaluate specific treatment modalities. Furthermore, it was not possible to extract the requisite information to estimate the $\mathrm{Cr}$ clearance from the chart, nor the exact time interval between initial insult to presentation to the ED. Because the medical records from which these data were abstracted were intended to support patient care and not clinical investigation activities, some charts did not contain information that would have contributed to this study (eg, serum phosphorous level and number of ampules given of sodium bicarbonate). Another issue is that a few charts were incorrectly coded as having a diagnosis of rhabdomyolysis. The "false positive" coding, although a rare occurrence, suggests that there could have been some "false negative" charts that were not included for analysis in this series, which would have created a source of information bias. In addition, by setting our search criteria using CPK greater than $1000 \mathrm{U} / \mathrm{L}$, we may have potentially missed many cases of rhabdomyolysis. However, we suspect that these cases are likely clinically insignificant for causing major complications such as ARF or need for HD. Another problem with generalizability was that almost all the patients assessed during the study period were male, many of which were minorities, which limits the generalizability of our results. The public hospital where this study was conducted has a locked prison ward and is a receiving facility for male prisoners from Riker's Island. Female inmates are sent to an alternate hospital facility. This probably accounts for much of the overrepresentation of men in our sample.

Regardless of how well a model can predict ARF and need for HD in patients admitted from the ED, one cannot definitively judge which patients are truly at low risk for complications on the basis of a single retrospective study. We therefore suggest that further prospective investigation be done to determine which subset of patients with rhabdomyolysis, after being given a standardized treatment (intravenous hydration, sodium bicarbonate, etc), can be safely discharged from the ED.

\section{Conclusion}

In a study of patients presenting with rhabdomyolysis to an urban ED setting, the main causes were cocaine abuse, exercise, and immobilization. A substantial proportion of patients with rhabdomyolysis developed the complications of ARF and required HD. We found that despite etiology or peak level of CPK, no patient developed ARF with an initial $\mathrm{Cr}$ less than 1.7. Except for initial serum $\mathrm{Cr}$ and BUN, clinical and laboratory factors were not reliable predictors for the development of ARF or need for HD in this case series. 


\section{References}

[1] Knochel JP. Rhabdomyolysis and myoglobinuria. Ann Rev Med 1982;33:435-43

[2] Knochel JP. Mechanisms of rhabdomyolysis. Curr Opin Rheumatol 1993;5:725-31.

[3] Salluzo RF. Rhabdomyolysis. In: Marx J, et al, editors. Rosen's Emergency Medicine: Concepts and Clinical Practice. 4th ed. St Louis (MO): Harcourt Brace; 1998. p. 2232-41.

[4] Koffler A, Freidler RM, Massry SG. Acute renal failure due to nontraumatic rhabdomyolysis. Ann Intern Med 1976;85:23-8.

[5] Olerud JE, Homer LD, Carroll HW. Incidence of acute exertional rhabdomyolysis. Arch Intern Med 1976;136:692-7.

[6] Gabow PA, Kaehny WD, Kelleher SP. The spectrum of rhabdomyolysis. Medicine 1982;61:141 - 52 .

[7] Chugh KS, Singhal PC, Nath IVS, et al. Acute renal failure due to non-traumatic rhabdomyolysis. Postgrad Med J 1979;55:386-92.

[8] Raifman MA, Berant M, Lenarsky C. Cold weather and rhabdomyolysis. J Pediatr 1978;93:970 - 1

[9] Patel R, Ansari A, Hughes JI. Myoglobinuric acute renal failure associated with phencyclidine abuse. West J Med 1979;131:244.

[10] Singhal PC, Chugh KS, Gulati DR. Myoglobinuria and renal failure after status epilepticus. Neurology 1978;28:200-1.

[11] Schiff HB, MacSerraigh ET, Kallmeyer JC. Myoglobinuria, rhabdomyolysis and marathon running. Q J Med 1978;47:463 - 72.

[12] Better OS, Stein JH. Early management of shock and prophylaxis of acute renal failure in traumatic rhabdomyolysis. NEngl J Med 1990;322: $825-9$.

[13] Richter RW, Challenor YB, Pearson J, et al. Acute myoglobinuria associated with heroin addiction. JAMA 1971;216:1172-6.

[14] Singhal P, Horowitz B, Qinones MC, et al. Acute renal failure following cocaine abuse. Nephron 1989;52:76-8.

[15] Counselman FL, McLaughlin EW, Kardon EM, Bhambhani-Bhavnani AS. Creatine phosphokinase elevation in patients presenting to the emergency department with cocaine-related complaints. Am J Emerg Med 1997;15:221-3

[16] Vanek VW, Dickey-White HI, Signs SA, et al. Concurrent use of cocaine and alcohol by patients treated in the emergency department. Ann Emerg Med 1996;28:508-14.

[17] Pesik NT, Otten EJ. Severe rhabdomyolysis following a viral illness: a case report and review of the literature. J Emerg Med 1996;14:425-8.

[18] Sofat N, Bell S, Turner J, Warrens AN. A case of acute renal failure and compartment syndrome after an alcoholic binge. J Accid Emerg Med 1999;16:296-8.
[19] Richards JR. Rhabdomyolysis and drugs of abuse. J Emerg Med 2000;19:51-6.

[20] Shanks ES, Muth CM. Diver with decompression injury, elevation of serum transaminase levels, and rhabdomyolysis. Ann Emerg Med 2001;37:533-6.

[21] Lewin JJ, Nappi JM, Taylor MH. Rhabdomyolysis with concurrent atorvastatin and diltiazem. Ann Pharmacother 2002;36:1546-9.

[22] Traub SJ, Hoffman RS, Nelson LS. The "Ecstasy" hangover: hyponatremia due to 3,4-methylenedioxymethamphetamine. J Urban Health 2002;79:549-55.

[23] Shimazu T, Yoshioka T, Nakata Y, et al. Fluid resuscitation and systemic complications in crush syndrome: 14 Hanshin-Awaji earthquake patients. J Trauma 1997;42:641-6.

[24] Bywaters EGL, Beall D. Crush injuries with impairment of renal function. BMJ 1941;1:427-32.

[25] Goldfarb DS, Chung S. The absence of rhabdomyolysis-induced renal failure following the world trade center collapse. Am J Med 2002;113: 260.

[26] Cadnapaphornchai P, Taher S, McDonald FD. Acute drug-associated rhabdomyolysis: an examination of its diverse renal manifestations and complications. Am J Med Sci 1980;280:66-72.

[27] Honda N. Acute renal failure and rhabdomyolysis. Kidney Int 1983;23:888-98.

[28] Ward MM. Factors predictive of acute renal failure in rhabdomyolysis. Arch Intern Med 1988;148:1553-7.

[29] Sinert R, Kohl L, Rainone T, Scalea T. Exercise-induced rhabdomyolysis. Ann Emerg Med 1994;23:1301-6.

[30] Brody SL, Wrenn KD, Wilber MM, Slovis CM. Predicting the severity of cocaine-associated rhabdomyolysis. Ann Emerg Med 1990;19:1137-43.

[31] Welch RD, Todd K, Krause GS. Incidence of cocaine-associated rhabdomyolysis. Ann Emerg Med 1991;20:154-7.

[32] Roth D, Alarcon FJ, Fernandez JA, et al. Acute rhabdomyolysis associated with cocaine intoxication. N Engl J Med 1988;319: $673-7$.

[33] Norris KC, Thornhill-Joynes M, Tareen N. Cocaine use and chronic renal failure. Semin Nephrol 2001;21:362-6.

[34] Bakir A, Dunea G. Renal disease in the inner city. Semin Nephrol 2001;21:334-45.

[35] Muckart DJJ, Moodley M, Naidu AG, et al. Prediction of acute renal failure following soft-tissue injury using the venous bicarbonate concentration. J Trauma 1992;33:813-7. 